\title{
A resposta de frequência cardíaca durante as competições de "mountain bike cross-country"
}

CDD. 20.ed. 796.022

796.6

\author{
Vitor Pereira COSTA \\ Fernando Roberto de OLIVEIRA**
}

${ }^{*}$ Centro de Educação

Superior da Região Sul, Universidade do Estado de Santa Catarina - Laguna.

**Universidade Federal

de Lavras.

\section{Resumo}

0 objetivo deste estudo foi verificar a resposta de frequência cardíaca (FC) durante as competições de "Cross-country" Olímpico (XCO). Quatorze "mountain bikers" foram separados em dois grupos: elite ( $n=6$; $26,5 \pm 3,6$ anos; $69,1 \pm 2,1 \mathrm{~kg} ; 174,0 \pm 1,2 \mathrm{~cm} ; 5,9 \pm 0,9 \% \mathrm{G} ; 9,0 \pm 1,3$ anos de treinamento) e amadores ( $\mathrm{n}=8 ; 25,6 \pm 7,7$ anos; $67,7 \pm 7,0 \mathrm{~kg} ; 175,5 \pm 5,5 \mathrm{~cm} ; 5,8 \pm 2,1 \% \mathrm{G} ; 8,3 \pm 5,7$ anos de treinamento). Os participantes foram submetidos a um teste progressivo para a identificação dos limiares metabólicos e seus respectivos valores de frequência cardiaca (FC) em cada zona de intensidade de esforço. Após intervalo mínimo de quatro dias os atletas da categoria elite foram avaliados através de monitores de FC durante a etapa brasileira da Copa do Mundo de XCO. Após 15 dias, todos os atletas foram avaliados no Campeonato Brasileiro de XCO. Os resultados indicaram que em ambas as competições, a média percentual da FC foi correspondente a 91-92 \% da FC ${ }_{\text {máx }}$ Nas competições, os atletas permaneceram durante diferentes tempos percentuais nas zonas de intensidade de esforço sendo 10,0-14,8\% no domínio leve; 23,1-30,1\% moderado e 55,1-66,9\% intenso. Assim, este estudo apresenta que as competições de XCO são realizadas em alta intensidade, principalmente após a largada.

Unitermos: "Mountain Bike"; Ciclismo; Frequência cardiaca.

\section{Introdução}

O "cross-country" olímpico (XCO) é uma das modalidades do "mountain bike" (MTB) realizada em trilhas estreitas e sinuosas denominadas "single tracks" ou em estradas de terra abertas, geralmente com a presença de erosóes, pedras, cascalhos, troncos, árvores e travessia em trechos com lama (PFeIFFER \& KRONISH, 1995). As competições de XCO são realizadas em circuito e consistem em um número definido de voltas, onde todos os participantes partem juntos em um único pelotão. A duração do evento está, em geral, entre $2 \mathrm{~h}$ para homens e $1 \mathrm{~h}$ e $45 \mathrm{~min}$ para as mulheres. As primeiras competiçôes de MTB foram disputadas na Califórnia nos EUA no início dos anos 80 , sendo oficializadas pela União Ciclística Internacional (UCI) apenas em 1990. Com o crescente número de praticantes, o XCO foi acrescentado ao programa oficial dos Jogos Olímpicos em Atlanta, 1996 (UCI, 2008). Nos últimos anos surgiu o interesse de pesquisadores na modalidade sendo que alguns estudos foram realizados em atletas e competições de XCO internacionais (COSTA,
Carminatti, Nakamura \& De-Oliveira, 2008; Costa \& De-Oliveira, 2008, 2009; Costa, Nakamura \& De-Oliveira, 2007; Impellizzeri, Marcora, Rampinini, Mognoni, \& Sassi, 2005a; ImpellizZeri, Rampinini, Sassi, Mognoni \& Marcora, 2005b; Impellizzeri, Sassi, Rodriguez-Alonso, Mognoni \& Marcora, 2002; LeE, Martin, Anson, Grundy \& Hahn, 2002; StaPelfeldT, SchWirtZ, SCHUMaChER \& Hillebrecht, 2004; Wilber, ZaWadzi, Kearney, Shannon \& DisALVO, 1997). No "ranking" mundial, o crescimento no número de participantes nas competições de XCO também acontece de forma significante sendo observado nas diversas categorias dos principais eventos internacionais nos últimos anos (UCI, 2008).

Como modalidade emergente, os profissionais envolvidos na preparação física de atletas têm dificuldades em encontrar referências de treinamento específicas avaliadas nesta modalidade. Neste sentido, para determinação da demanda fisiológica durante o exercício é comumente utilizada a simples resposta da frequência 
cardíaca (FC). A resposta da FC durante o exercício pode ser considerada como reflexo da demanda energética, pois está diretamente relacionado com o aumento do consumo de oxigênio $\left({ }^{\top} \mathrm{O}_{2}\right)$ pela musculatura esquelética envolvida. Assumindo uma relação linear entre o $\mathrm{VO}_{2}$ e a FC durante o esforço, talvez seja possível utilizá-la como medida da taxa metabólica durante o exercício físico mesmo em modalidades intermitentes como o XCO. Como pode ser considerada de fácil medida, a FC é um dos indicadores de intensidade de esforço frequentemente usados em esportes como o ciclismo (Boulay, 1995). Assim, os monitores de FC aparecem como uma opção acessível e uma ferramenta muito útil em esportes de capacidade aeróbia. Devido à precisão de medidas, grande capacidade de armazenar informações e funções práticas; muitos atletas têm comprovado suas vantagens nas competições e/ ou treinamento (JeUKENDRUP \& VAN Diemen, 1998).

$\mathrm{Na}$ tentativa de apresentar a demanda fisiológica das provas internacionais de MTB, IMPELLIZZERI et al. (2002) descreveram a resposta da FC dos atletas durante as provas de XCO. Os resultados apresentaram que a intensidade corresponde a aproximadamente $90 \%$ da frequência cardíaca máxima $\left(\mathrm{FC}_{\text {máx }}\right)$. Além disso, os autores utilizaram o modelo de zonas de intensidade de esforço aeróbio baseados na FC correspondente aos limiares metabólicos, onde verificaram que os atletas permanecem $31 \%$ do tempo total de competição acima do "onset blood lactate accumulation" (OBLA). Sendo assim, os praticantes devem suportar cargas internas no domínio intenso onde as exigências frequentemente, estão próximas aos indicadores de potência e capacidade aeróbia. A determinação da resposta de FC dos ciclistas durante as provas de XCO se torna uma referência importante para técnicos e treinadores, na tentativa de quantificar o esforço dos treinos de acordo com a exigência da competição. Para nosso conhecimento, apenas um estudo investigou a intensidade de esforço através da resposta da FC a que os atletas de MTB foram submetidos durante as competiçōes de XCO (IMPELlizzeri et al., 2002). Assim, o objetivo desse estudo é analisar e quantificar a intensidade de esforço através da resposta da FC dos ciclistas brasileiros durante as competições de XCO.

\section{Métodos}

Os atletas foram selecionados a partir de contatos prévios via telefone, correio eletrônico e encontros em competiçōes. Neste contexto, participaram do estudo 14 "mountain bikers" que disputam campeonatos estaduais e nacionais. No período em que foi realizado o experimento, os atletas apresentaram o registro das federaçôes em diferentes estados: MG $(\mathrm{n}=5), \mathrm{SC}(\mathrm{n}$ $=5), R J(n=3), M S(n=1)$, sendo classificados em diferentes categorias nos campeonatos nacionais: elite $(\mathrm{n}=6)$, júnior $(\mathrm{n}=1)$, sub $23(\mathrm{n}=3)$, sub $30(\mathrm{n}=1) \mathrm{e}$ master $(\mathrm{n}=3)$. Em relação ao período de treinamento, os atletas relataram estar na fase competitiva. Assim, o grupo de 14 atletas foi separado em dois grupos: 1) ciclistas que competem na categoria elite e 2) ciclistas que competem nas demais categorias, sendo denominados no presente estudo de amadores (TABELA 1).

Este estudo foi aprovado pelo Comitê de Ética da Universidade do Estado de Santa Catarina $\left(\mathrm{n}^{0}\right.$ 017/05). Após ler e assinar o termo de consentimento informado, os participantes foram submetidos à avaliação em duas etapas: 1) antropométrica e aeróbia, respectivamente; 2) avaliação durante duas competições de XCO. Todos os testes laboratoriais foram realizados no Laboratório de Avaliação Morfofuncional (LAPEM), no Centro de Educação Física, Fisioterapia e Desportos (CEFID), na Universidade do Estado de Santa Catarina (UDESC). Após intervalo mínimo de quatro dias, os atletas foram avaliados através de monitores de FC (Polar®: modelo S610i), durante a sexta etapa da Copa do Mundo de MTB. Esta competição foi realizada no circuito de XCO, localizado no Parque Unipraias, na cidade de Balneário Camboriú - SC, no dia 3 de julho de 2005. Após duas semanas, os atletas foram avaliados durante o Campeonato Brasileiro de MTB, realizado no Parque das Laranjeiras, na cidade de Três Coroas - RS, no dia 17 de julho de 2005. Todos os atletas foram instruídos para que evitassem a ingestão de alimentos sólidos no período de $3 \mathrm{~h}$ prévio a realização dos testes laboratoriais, com permissão para a ingestão de líquidos sem excessos. Foram recomendados somente a praticarem atividades físicas de baixa intensidade nos dias antecedentes ao experimento e/ou treinos em baixa intensidade nas $24 \mathrm{~h}$ anteriores as competiçōes (TAYLOR, Wang, Rowell \& BlomQVist, 1963). 
TABELA 1 - Características dos sujeitos (média \pm desvio padrão).

\begin{tabular}{|c|c|c|}
\hline Variáveis & Elite $(n=6)$ & Amadores $(n=8)$ \\
\hline Idade (anos) & $26,5 \pm 3,6$ & $25,6 \pm 7,7$ \\
\hline Massa corporal (kg) & $69,1 \pm 2,1$ & $67,7 \pm 7,0$ \\
\hline Estatura $(\mathrm{cm})$ & $174,8 \pm 1,2$ & $175,5 \pm 5,5$ \\
\hline Percentual de gordura (\%) & $5,9 \pm 0,9$ & $5,8 \pm 2,1$ \\
\hline Anos de treinamento & $9,0 \pm 1,3$ & $8,3 \pm 5,7$ \\
\hline $\mathrm{W}_{\text {máx }}$ (watts) & $349,2 \pm 15,6$ & $335,0 \pm 27,8$ \\
\hline $\mathrm{W}_{\text {máx.kg-1 }}\left(\right.$ watts. $\left.\mathrm{kg}^{-1}\right)$ & $5,1 \pm 0,2$ & $5,0 \pm 0,2$ \\
\hline $\mathrm{y}^{\top} \mathrm{O}_{2 \mathrm{máx}}\left(1 . \mathrm{min}^{-1}\right)$ & $4,8 \pm 0,2$ & $4,6 \pm 0,7$ \\
\hline $\mathrm{W}^{\prime} \mathrm{O}_{2 \text { máx.kg-1 }}\left(\mathrm{ml} \cdot \mathrm{kg}^{-1} \cdot \mathrm{min}^{-1}\right)$ & $69,8 \pm 3,5$ & $68,5 \pm 4,8$ \\
\hline $\mathrm{FC}_{\text {máx }}(\mathrm{bpm})$ & $187 \pm 5^{*}$ & $195 \pm 7$ \\
\hline$[\mathrm{La}]$ pico $\left(\mathrm{mmol} . \mathrm{l}^{-1}\right)$ & $10,9 \pm 2,3$ & $12,4 \pm 2,8$ \\
\hline $\mathrm{WLL}_{1}$ (watts) & $265 \pm 20^{*}$ & $235 \pm 31$ \\
\hline $\mathrm{WLL}_{2}$ (watts) & $305 \pm 19^{*}$ & $274 \pm 33$ \\
\hline $\mathrm{FCLL}_{1}(\mathrm{bpm})$ & $157 \pm 11$ & $162 \pm 13$ \\
\hline $\mathrm{FCLL}_{2}(\mathrm{bpm})$ & $174 \pm 11$ & $173 \pm 10$ \\
\hline
\end{tabular}

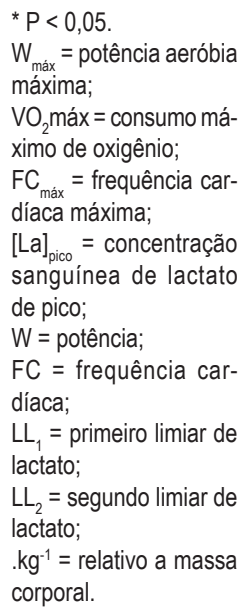

\section{Avaliação laboratorial}

Para estimativa do percentual de gordura $(\% \mathrm{G})$, as dobras cutâneas foram mensuradas nas regiōes do tórax, abdômen e coxa, conforme as descrições do protocolo de três dobras, proposto por JACKSON e POLLOCK (1978) e segundo a padronização de LOHMAN, Roche e Matorell (1998). A avaliação aeróbia foi realizada na própria bicicleta do atleta acoplada ao ciclo-simulador (CompuTrainer ${ }^{\mathrm{TM}}$ RacerMate ${ }^{\circledR} 8000$, Seattle WA). O aparelho mensura a potência produzida e rotaçôes por minuto (rpm). Antes de iniciar o teste, os participantes realizaram aquecimento com carga de 70 W e duração de $8 \mathrm{~min}$, para a posterior calibração do ergômetro. Em seguida, o teste foi iniciado com carga de $100 \mathrm{~W}$ e incrementos de $30 \mathrm{~W}$ a cada $3 \mathrm{~min}$, até exaustão. Os atletas mantiveram frequência no pedal entre 90 e 110 rpm durante todo o teste, sendo que a relação coroa/cassete permaneceu fixa, correspondente a 44/17. A falta de sustentação da cadência no pedal e/ ou exaustão voluntária, foram os critérios utilizados para a interrupção do teste. Quando a carga do estágio não foi completada, a potência aeróbia máxima $\left(\mathrm{W}_{\text {máx }}\right)$ foi identificada segundo o método de KUIPERS, VERSTAPPEN, Geurten e Van Kranenburg (1985):

$$
\mathrm{W}_{\text {máx }}=\mathrm{Wf}+(\mathrm{t} / 180 \times 30)
$$

onde Wf é a carga em W do último estágio, t é o tempo em segundos do estágio incompleto, 180 é o tempo em segundos proposto para cada duração de estágio e $30 \mathrm{~W}$ é o valor do incremento das cargas.

A FC foi monitorada durante todo o teste, através de um monitor de FC (Polar®: modelo S610i). Durante os 30 segundos finais de cada estágio, foram coletados $25 \mu \mathrm{l}$ de sangue arterializado no lóbulo da orelha, previamente hiperemiada com Finalgon $\AA$, para análise das concentrações sanguíneas de lactato [La]. As [La] foram imediatamente analisadas no lactímetro da marca Yellow Springs ${ }^{\circledR}$ (modelo 1500).

Os limiares de lactato (LL) foram identificados individualmente a partir do comportamento da curva das [La] em função da intensidade do esforço, sendo expressos nos valores de FC a partir da interpolação entre dois pontos adjacentes. $\mathrm{O}$ método de identificação do primeiro LL $\left(\mathrm{LL}_{1}\right)$ foi proposto por Hagberg e CoYle (1983), que definiram como sendo a intensidade de exercício que determina um

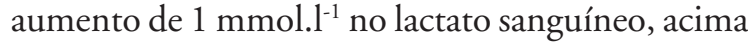
dos valores da linha de base. O segundo LL $\left(\mathrm{LL}_{2}\right)$ foi definido a partir da identificação do OBLA, que sugere a concentração fixa de $4 \mathrm{mmol}^{-1}{ }^{-1}$ (SJOdin \& JACOBS, 1981) Para quantificar o tempo despendido em diferentes intensidades de esforço durante as competiçôes de XCO (\% de tempo em cada domínio fisiológico), foram identificados os respectivos valores de FC correspondentes aos LL no teste progressivo realizado previamente no laboratório. 
Assim, foram classificados três domínios fisiológicos baseados em modelo prévio (GILMAN, 1996) e sendo aqui chamado de: leve (abaixo de $\mathrm{LL}_{1}$ ), moderado (entre $\mathrm{LL}_{1}$ ) e $\mathrm{LL}_{2}$ e intenso (acima de $\mathrm{LL}_{2}$ ).

$\mathrm{O}_{\mathrm{WO}}$ foi monitorado durante todo o teste, sendo que os valores registrados foram de apenas 10 atletas devido a problemas de calibração e funcionamento inadequado do aparelho. As trocas gasosas foram registradas a cada 20 segundos, através de uma máscara conectada a um analisador de gases de circuito aberto Aerosport KB1-C (Aerosport ${ }^{\circledR}$, Inc., Ann Arbor, $\mathrm{MI}$ ) onde os indivíduos ventilaram durante todo teste. King, Mclaughin, Howley, Bassett Junior e AINSWORTH (1999) estudaram a validade do analisador de gases, em situaçôes de repouso e intensidades correspondente a 50,100, 150, 200 e $250 \mathrm{~W}$. Os resultados sugerem que as medidas de $\dot{\mathrm{V}}_{2}$, realizadas pelo analisador Aerosport KB1-C, são aceitáveis entre valores de 1,5 a 3,5 $1 \cdot \mathrm{min}^{-1}$. Os dados de ${ }^{\mathrm{W}} \mathrm{O}_{2}$ foram plotados em função da potência, sendo que o maior valor atingido no teste foi considerado o $\mathrm{WO}_{2 \text { pico }}$.

\section{Avaliação durante as competições de XCO}

Os atletas da categoria elite foram avaliados durante duas competições de XCO, válidas pela sexta etapa da Copa do Mundo de MTB e a etapa única do Campeonato Brasileiro, sendo que, as categorias amadoras disputaram apenas o Campeonato Brasileiro de XCO. Foi encaminhado um ofício a Federação Estadual de Ciclismo com todos os procedimentos a serem executados, ficando a organização e os atletas livres de qualquer responsabilidade em relação a esta pesquisa.

O registro da intensidade de esforço das competições foi realizado através da utilização de monitores de FC que mensuram os batimentos cardíacos durante as

\section{Resultados}

As características dos ciclistas estão apresentadas na TABELA 1. Das variáveis analisadas houve diferença significativa entre os grupos nos valores de potência em que os LL foram identificados, sendo verificados valores maiores para os atletas da categoria elite $(p<0,05)$. No entanto, os valores de absolutos de FC nos LL permaneceram semelhante. Também houve diferenças significativas na $\mathrm{FC}_{\text {máx }}$. em que os valores foram altos no atletas amadores $(\mathrm{p}<0,05)$. competiçóes em intervalos de 5 s. Aproximadamente uma hora antes do início do evento, os monitores de FC foram colocados nos atletas. Auxiliares da pesquisa responsáveis individualmente pelos atletas registraram o tempo de cada volta através de cronômetros. Após o término da competição, os auxiliares ficaram responsáveis por desligar os monitores de FC e transferir os dados para o microcomputador.

Dos atletas avaliados na Copa do Mundo de XCO, apenas um conseguiu completar o número de voltas determinado pela organização, sendo que, quatro completaram a prova com uma volta a menos que o pelotão principal e um atleta completou a prova com duas voltas a menos em relação aos primeiros. Além disso, um atleta abandonou a competição na segunda volta, devido a um acidente durante o percurso. É importante ressaltar que os atletas brasileiros que não completaram o número definido de voltas na Copa do Mundo de XCO foram obrigados a parar durante a competição devido aos critérios pré-estabelecidos pela União Ciclística Internacional (UCI). Resumidamente, o regulamento determina que o atleta que permanecer a uma distância (tempo de prova) correspondente a $80 \%$ mais lento que o líder da prova será obrigado a se retirar da competição.

\section{Tratamento estatístico}

A estatística descritiva foi utilizada em valores de média e desvio padrão (dp). Após a determinação da distribuição da normalidade dos dados, foi escolhida estatística paramétrica para comparação das variáveis morfofisiológicas entre os atletas das categorias elite e amadores, sendo utilizado o teste t de "student". Para a análise estatística foi escolhido o "software" SPSS ${ }^{\circ}$ 11.0 e o nível de significância adotado foi $\mathrm{p}<0,05$.

A grande diferença entre os dois eventos foram as condições climáticas. O Campeonato Brasileiro de $\mathrm{XCO}$ foi realizado em um dia nublado com temperatura de $7^{\circ} \mathrm{C}$ e umidade relativa do ar de $70 \%$, sendo a Copa do Mundo disputada em dia ensolarado com temperatura de $28^{\circ} \mathrm{C}$ e umidade relativa do ar de $55 \%$.

A resposta da FC durante as competiçóes revelou que os participantes permaneceram em média durante os eventos aproximadamente a $91-92 \% \mathrm{da}$ $\mathrm{FC}_{\text {máx }}$ (FIGURA 1). 


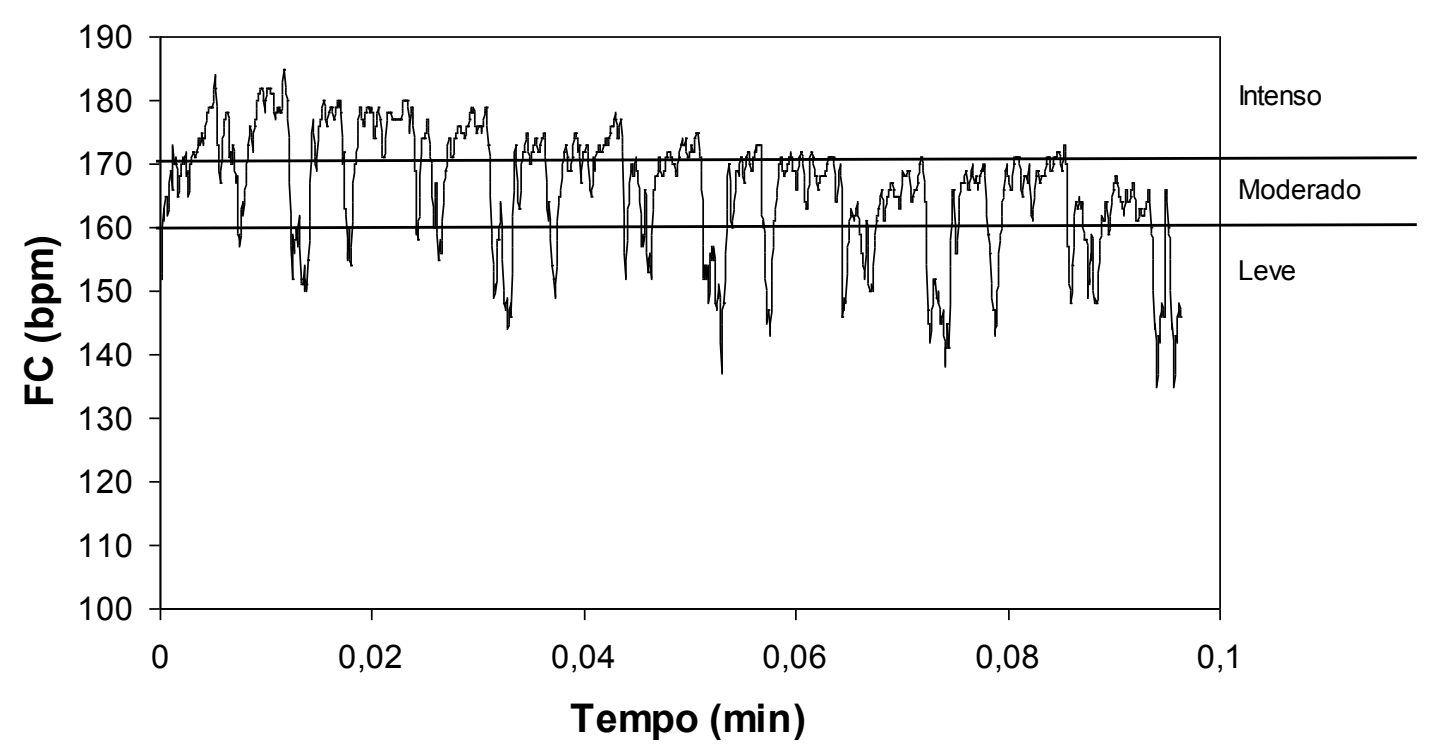

A linha reta superior representa FC em segundo limiar de lactato e a linha reta inferior representa a FC em primeiro limiar de lactato.

FIGURA 1 - Exemplo individual da FC na categoria Elite durante a Copa de Mundo de XCO.

Os resultados indicaram que grande parte das competiçōes, independente da categoria, é realizada no domínio fisiológico intenso, sendo pequena a contribuição do domínio moderado e insignificante a participação do domínio leve (FIGURA 2).

Em relação à $\mathrm{FC}$ média durante as competiçōes de XCO, de uma forma geral e independente da categoria, percebe-se uma redução durante as provas (FIGURA 3).

Aparentemente, o tempo de cada volta aumentou ao longo das voltas, em ambas as competições, sendo que em observações individuais os atletas que se destacaram, apresentam melhor regularidade durante a competição (FIGURA 4).
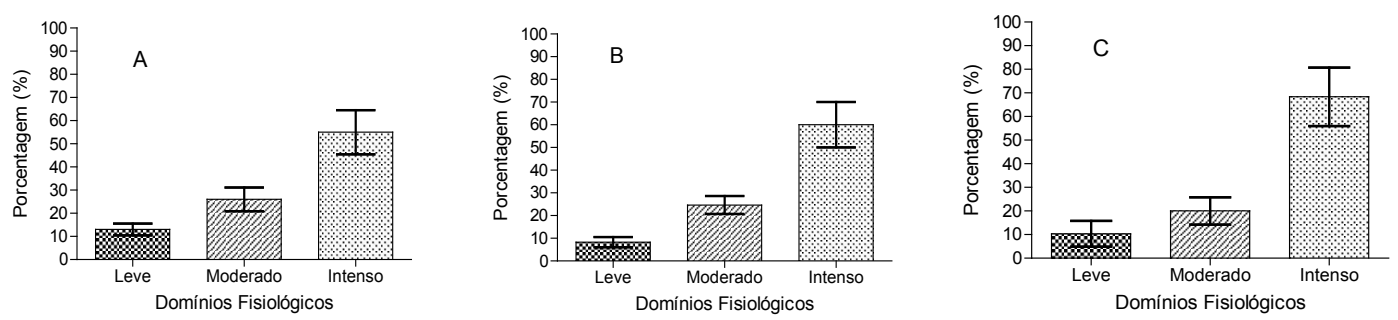

FIGURAS 2 e 3

$A=$ Campeonato Brasileiro (Elite);

$\mathrm{B}=$ Campeonato Brasileiro (Amadores);

$\mathrm{C}=$ Copa do Mundo (Elite).

Os dados estão representados em média e erro padrão da média.

FIGURA 2 - Porcentagens do tempo total durante competições de XCO, nos diferentes domínios fisiológicos.
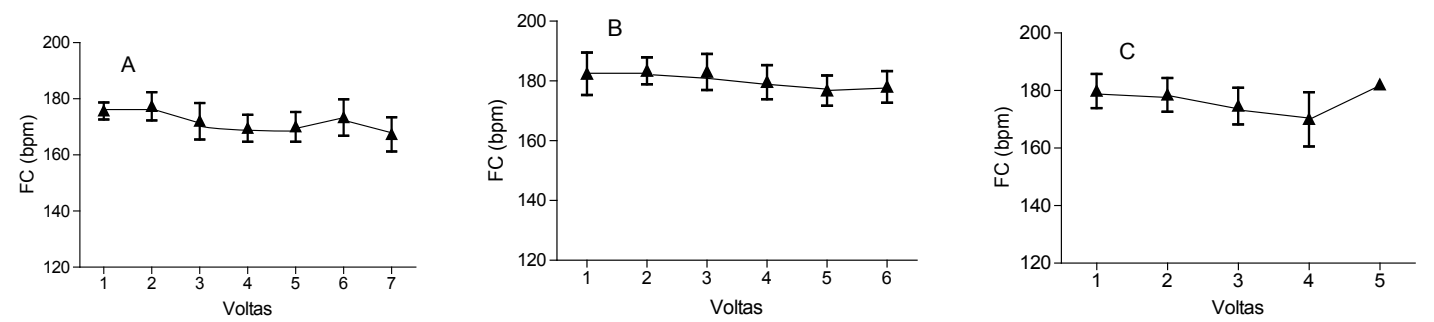

FIGURA 3 - FC média em cada volta, durante o Campeonato Brasileiro e a Copa do Mundo de XCO. 
$A=$ Campeonato Brasileiro (Elite);

$\mathrm{B}=$ Campeonato Brasileiro (Amadores);

C = Copa do Mundo (Elite).

Os dados estão representados em média e erro padrão da média.
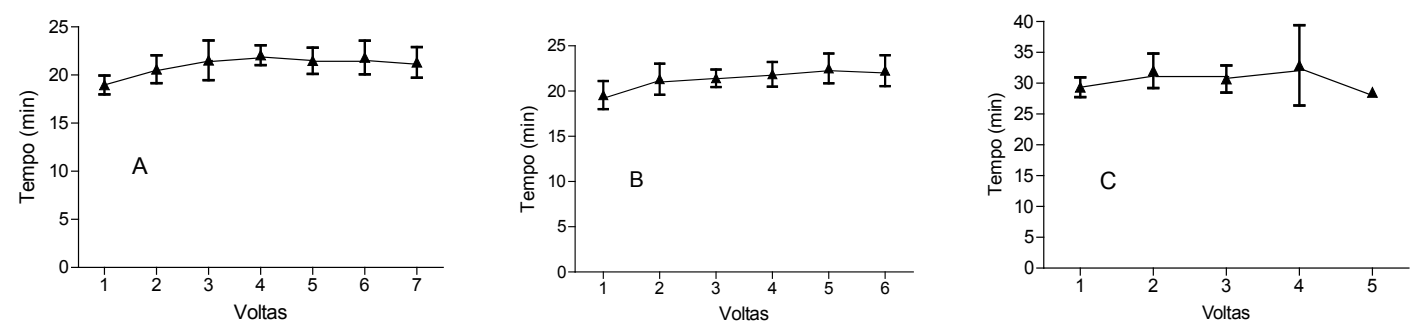

FIGURA 4 - Tempo de cada volta durante o Campeonato Brasileiro e a Copa do Mundo de XCO.

\section{Discussão}

A resposta da FC dos ciclistas durante as competiçóes de MTB XCO indica que os atletas foram submetidos à alta exigência fisiológica onde os valores ficaram em média próximos a 91-92\% da $\mathrm{FC}_{\text {máx }} \mathrm{du}-$ rante aproximadamente $125 \mathrm{~min}$ de prova caracterizando um esforço de alta intensidade e longa duração.

De acordo com estudo prévio realizado por IMPELLIZZERI et al. (2002), que avaliaram atletas italianos de MTB em diferentes circuitos de XCO, a resposta da FC indica valores médios de $90 \%$ da $\mathrm{FC}_{\text {máx }}$ nas competiçóes. STAPELFELDT et al. (2004) também avaliaram atletas alemães de MTB em 15 circuitos de $\mathrm{XCO}$ diferentes, sendo que a intensidade média do

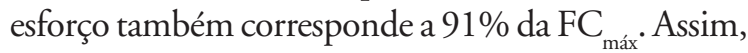
verifica-se que a média percentual da FC avaliada durante o Campeonato Brasileiro e na Copa do Mundo de XCO em atletas amadores e profissionais está de acordo com a literatura internacional.

Além da potência mecânica nos membros inferiores que produzem a elevação da FC, acredita-se que existem outros fatores observados durante as provas de $\mathrm{XCO}$ que também refletem nos altos valores de FC encontrados nos participantes. Dentre eles, destacam-se algumas características naturais do esporte que aumentam a resistência do atrito durante a pedalada, como a utilização de pneus largos e com travas, terrenos bastante irregulares e constantes subidas e descidas íngremes, todos esses fatores também refletem na menor velocidade média atingida nas pistas (IMPELLIZZERI et al., 2002). Além disso, no XCO não é possível que os "mountain bikers" percorram durante parte do percurso juntos em um único pelotão. Os longos trechos em "single tracks" são estreitos e só permitem que um atleta passe de cada vez. Assim, a situação de "vácuo" não pode ser formada e o ciclista não pode ser ajudado por sua equipe como ocorre no ciclismo (IMPELLIZZERI et al., 2002).
Outro fator que ajuda a manter a FC elevada é que durante as provas de XCO ocorrem repetidas e intensas contrações isométricas em membros superiores e inferiores que são necessárias para a absorção de impactos e das vibrações causadas pelo terreno, principalmente observado nas descidas (IMPELlizzeri et al., 2002). Com isso, especula-se que as açôes musculares isométricas podem contribuir para a manutenção elevada da FC nos circuitos sendo destacado nos trechos de descidas. $\mathrm{Na}$ tentativa de diminuir o impacto e o esforço para o ciclista, as fábricas oferecem diferentes modelos de suspensões. Entretanto, em nosso estudo todos os atletas utilizaram suspensões dianteiras de padrão competitivo e os valores de FC permaneceram altos de acordo com a literatura internacional.

A estratégia utilizada pelos atletas de XCO também pode ajudar a explicar as características da intensidade de esforço observado nas competições. Corroborando com nosso estudo, IMPELLIZZERI et al. (2002) verificaram que os valores máximos de FC estão na primeira volta logo após a largada (FIGURA 1). Sem dúvida, a largada constitui um dos momentos mais importantes do evento, pois logo após, as pistas se estreitam em longos "single tracks" onde é difícil de realizar ultrapassagens. STAPELFELDT et al. (2004) encontraram que os "mountain bikers" atingem valores de potência acima da $\mathrm{W}_{\text {máx }}$ logo após a largada levando o organismo uma exigência fisiologia intensa onde rapidamente o ciclista atinge altos valores de $\mathrm{FC} \mathrm{em}$ resposta a demanda imposta pela largada.

$\mathrm{O}$ monitoramento das competições de XCO também revela que durante as consecutivas voltas, a FC média diminui em ambos os eventos. A diminuição da FC média pode ser explicada em parte, pela possível queda de rendimento e consequente diminuição na potência produzida pelos ciclistas. As 
fontes bioenergéticas responsáveis pela manutenção da intensidade de esforço em valores próximos a 90\% $\mathrm{FC}_{\text {máx }}$ são predominantemente utilizadas a partir de carboidratos, sendo que ao longo da prova os sintomas de fadiga podem ser percebidos a partir da depleção energética. O tempo total de cada volta aumentou em ambas as competições, o que indiretamente fortalece tais especulações sobre a queda de rendimento. Em observaçôes individuais percebe-se que os primeiros colocados tendem a manter constante o tempo de cada volta ao longo da competição e provavelmente discreta diminuição da potência mecânica. É interessante ressaltar que não foi observado em nenhum atleta, o aumento da FC durante as competições de XCO. Esse fenômeno é conhecido como desvio cardiovascular e pode ser iniciado a partir da desidratação e consequente falha nos mecanismos de termorregulação do atleta. Nossos resultados são corroborados pelo estudo de IMPELLIZZERI et al.(2002) que também verificaram queda da FC ao longo das provas e ausência de desvio cardiovascular nos ciclistas.

O comportamento da FC durante as competições indica a extensão em que os atletas permaneceram nos domínios fisiológicos pré-estabelecidos. Nos dois eventos, verifica-se a insignificante contribuição do domínio leve (10,0-14,8\%) e moderado (23,1-30,1\%), sendo predominante a participação do domínio intenso $(55,1$ $66,9 \%$ ). IMPELLIZZERI et al. (2002) utilizaram o modelo de zonas de esforço para quantificar o tempo que os atletas permaneciam em cada domínio fisiológico durante as competições de XCO. Os autores verificaram que durante as provas os ciclistas permanecem a $18 \%$, $51 \%$ e $31 \%$ do tempo total de prova, nos domínios leve, moderado e intenso, respectivamente. Nossos resultados indicam discordância no tempo percentual permanecido nos diferentes domínios fisiológicos. Apesar disso, o tempo total das provas e a quilometragem foram semelhantes aos estabelecidos pelas regras da UCI, contribuindo para uma distribuição proporcional equivalente do esforço nas provas.

Em estudo mais recente STAPELFELDT et al. (2004) utilizaram o modelo de zonas de intensidade de esforço através da análise da resposta da potência durante as competições de XCO. Neste estudo, os autores classificaram quatro zonas de intensidade de esforço: abaixo da potência em $\mathrm{LL}_{1}\left(\mathrm{LL}_{1}-39 \%\right)$, entre $\mathrm{LL}_{1} \mathrm{e}$ $\mathrm{LL}_{2}(19 \%)$, entre $\mathrm{LL}_{2} \mathrm{e} \mathrm{W}_{\text {máx }}$ (20\%), e acima da $\mathrm{W}_{\text {máx }}$ (22\%). Os resultados são distintos, pois os autores avaliaram os domínios fisiológicos através da resposta da potência e não da FC, além dos métodos de identificação de limiares metabólicos serem diferentes. Recentemente, Vogt, Heinrich, Schumacher, Blum,
RoeCKeR, DickHUTH e SCHMiD (2006) compararam o modelo de zonas de intensidade de esforço a partir da utilização de monitores de FC e dinamômetros portáteis, durante uma competição de seis dias no ciclismo de estrada. Os resultados da resposta da FC indicam que os atletas permanecem mais tempo nos domínios abaixo de $\mathrm{LL}_{1}$ e acima de $\mathrm{LL}_{2}$, se comparado com a potência $\left(<\mathrm{LL}_{1}=58 \%\right.$ e $38 \%$ vs $>\mathrm{LL}_{2}=28 \%$ e $24 \%$ ), respectivamente. Assim, devido às grandes oscilações de terreno no XCO, deve-se ter cautela ao estimar e/ou interpretar, os valores de potência e os percentuais de tempo despendido em cada domínio fisiológico através da FC.

Um dos achados interessantes do estudo de STAPELFELDT et al. (2004) durante as provas de XCO é o comportamento constante da FC e oscilante da potência produzida. Fica claro que no XCO, a potência produzida pelos membros inferiores não é constante como nas provas de contra-relógio no ciclismo de estrada, no entanto, a exigência cardíaca é semelhante. Além disso, o grupo alemão descreveu que o perfil da potência produzida durante a prova sofreu grande oscilação dependendo do trecho no percurso. Em subidas curtas, os valores da potência ultrapassaram os valores da $\mathrm{W}_{\text {máx }}$ registrada previamente no laboratório, enquanto que nas descidas mesmo com a potência sendo nula, a FC se mantém elevada. Estes achados sustentam as inferências feitas anteriormente sobre as contrações musculares isométricas na manutenção elevada da FC em situações de baixa ou ausência de carga de esforço nos membros inferiores.

$\mathrm{Na}$ tentativa de quantificar a exigência fisiológica no MTB, Dal Monte e Faina (1999) analisaram a resposta da FC e coletaram amostras de [La] nos atletas em cada volta durante uma simulação de competição de XCO. Os resultados indicaram que a FC se eleva no início e permanece constante durante a prova, sendo que nas primeiras voltas e até aproximadamente $40 \mathrm{~min}$ de competição, os atletas atingem elevados valores de [La] de 10-11 mmol.1 ${ }^{-1}$. Durante a competição de XCO, as [La] regridem gradativamente, e ao final da prova, os valores atingem 5-4 mmol. ${ }^{-1}$. Apesar dos autores terem realizado a simulação de uma competição, os resultados de FC estão de acordo com o nosso estudo e com as especulações prévias, indicando alta exigência fisiológica durante as primeiras voltas e queda na intensidade ao final da prova. Assim, percebe-se que as competições de XCO são predominantemente aeróbias, com grande participação do metabolismo anaeróbio. 


\title{
Conclusão
}

Nosso estudo apresenta que a resposta da FC durante as competições de XCO tem um comportamento elevado e relativamente constante durante todo o evento. Durante o início das provas os atletas atingem altos valores de $\mathrm{FC}$ que tendem a diminuir ao longo da competição. $\mathrm{O}$ conhecimento sobre a intensidade de esforço realizada durante as competições de XCO pode ser utilizado por treinadores e atletas para elaboração dos programas de treinamento de acordo com a realidade esportiva. Além disso, nosso estudo também destaca a utilização de monitores de FC para o controle da intensidade de esforço durante treinos e/ou competições de
MTB. A fácil disponibilidade, precisão de medidas e relação custo/benefício favorece a sua utilização no XCO. No entanto, vale ressaltar que a FC é facilmente afetada por fatores ambientais, hormonais, farmacológicos e psicológicos; e em diversas situações como treinos e competições pode haver dissociação entre a FC e a demanda metabólica que está sendo realizada na atividade. Assim, o XCO é um esporte caracterizado por elevada exigência fisiológica sendo que a resposta da FC dos atletas nas competições avaliadas indica que o esporte é disputado em grande parte na intensidade próxima ao domínio fisiológico intenso.

\begin{abstract}
Heart rate response during mountain bike cross-country races

The aim of this study was to verify and describe the intensity profile of cross-country mountain-biking races using heart rate $(H R)$ recorded during races. Fourteen mountain bikers participated in two groups: elite $(n=6 ; 26.5 \pm 3.6$ years old; $69.1 \pm 2.1 \mathrm{~kg} ; 174.0 \pm 1.2 \mathrm{~cm} ; 5.9 \pm 0.9 \%$ BF; $9.0 \pm 1.3$ years of training) and amateurs $(n=8 ; 25.6 \pm 7.7$ years; $67.7 \pm 7.0 \mathrm{~kg} ; 175.5 \pm 5.5 \mathrm{~cm} ; 5.8 \pm 2.1 \% \mathrm{BF} ; 8.3 \pm 5.7$ years of training). Each cyclist was submitted to an incremental exercise test to determine the metabolic thresholds and the HR values at each threshold. After four days, only the athletes of elite category were tested during Brazilian round of XCO World Cup. Two weeks later both groups were evaluated in XCO Brazilian National Championship. The results showed that HR averaged $91-92 \%$ of $\mathrm{HR}_{\max }$. The exercise intensity zones showed different percentage of time spent during the races (10.0-14.8\% easy; 23.1$30.1 \%$ moderate; $55.1-66.9 \%$ hard). This study shows that cross-country events are conducted at very high intensity, especially at the start of the race.
\end{abstract}

UNITERMS: Mountain bike; Cycling; Heart rate.

\section{Referências}

BOULAY, M.R. Physiological monitoring of elite cyclists. Sports Medicine, Auckland, v.20, n.1, p.1-11, 1995.

COSTA, V.P.; CARMINATTI, L.J.; NAKAMURA, F.Y.; DE-OLIVEIRA, F.R. Morph-Physiological similarities between road cyclists and mountain bikers. L'Aquila. Italian Journal of Sports Sciences, L'Aquila, n.1, p.1-11, 2008.

COSTA, V.P.; DE-OLIVEIRA, F.R. Physiological variables to predict performance in mountain bike cross-country races. Journal of Exercise Physiology-online, Duluth, v.11, n.6, p.14-24, 2008.

Aspectos morfológicos e fisiológicos no ciclismo de estrada e mountain bike cross country. Revista da Educação

Física do Exército, Rio de Janeiro, v.145, p.11-20, 2009.

COSTA, V.P.; NAKAMURA, F.Y.; DE-OLIVEIRA, F.R. Aspectos fisiológicos e de treinamento de mountain bikers brasileiros.

Revista da Educação Física do Exército, Rio de Janeiro, v.136, p.5-11, 2007.

DAL MONTE, A.; FAINA, M. Valutazione delli atleta. Torino: UTET, 1999.

GILMAN, M.B. The use of heart rate to monitor the intensity of endurance training. Sports Medicine, Auckland, v.21, n.2, p.73-79, 1996. 
HAGBERG, J.M.; COYLE, E.F. Physiological determinants of endurance performance as studied in competitive racewalkers. Medicine and Science in Sports and Exercise, Madison, v.15, p.287-9, 1983.

IMPELLIZZERI, F.M.; MARCORA, S.M.; RAMPININI, E.; MOGNONI, P.; SASSI, A. Correlations between physiological variables and performance in high level cross country off road cyclists. British Journal of Sports Medicine, London, v.39, p.747-51, 2005a.

IMPELLIZZERI, F.M.; RAMPININI, E.; SASSI, A.; MOGNONI, P.; MARCORA S. Physiological correlates to off-road cycling performance. Journal of Sports Sciences, London, v.23, p.41-7, 2005b.

IMPELLIZZERI, F.M.; SASSI, A.; RODRIGUEZ-ALONSO, M.; MOGNONI, P.; MARCORA, S. Exercise intensity during off-road cycling competitions. Medicine and Science in Sports and Exercise, Madison, v.34, p.1808-13, 2002.

JACKSON, A.L.; POLLOCK, M.L. Generalized equations for prediction body density of men. British Journal of Nutrition, Cambridge, v.40, p.497-504, 1978.

JEUKENDRUP, A.; VAN DIEMEN, A. Heart rate monitoring during training and competition in cyclists. Journal of Sports Sciences, London, v.16, p.S91-S99, 1998.

KING, G.A.; McLAUGHLIN, J.E.; HOWLEY, E.T.; BASSETT JUNIOR, D.R.; AINSWORTH, B.E. Validation of Aerosport KB1-C portable metabolic system. International Journal of Sports Medicine, Suttgart, v.20, n.5, p.304-8, 1999. KUIPERS, H.; VERSTAPPEN, F.T.J.; GEURTEN, P.; VAN KRANENBURG, G. Variability of aerobic performance in laboratory and its physiologic correlates. International Journal of Sports Medicine, Suttgart, v.6, p.197-201, 1985.

LEE, H.; MARTIN, D.T.; ANSON, J.M.; GRUNDY, D.; HAHN, A.G. Physiological characteristics of successful mountain bikers and professional road cyclists. Journal of Sports Science, Wallsall, v.20, p.1001-8, 2002.

LOHMAN, T.G.; ROCHE, A.F.; MATORELL, R. Anthropometric standardization reference manual. Champaign: Human Kinetics, 1998.

PFEIFFER, R.P.; KRONISH, R.L. Off-road cycling injuries: an overview. Sports Medicine, Auckland, v.19, n.5, p.311-25, 1995. SJODIN, B.; JACOBS, I. Onset blood lactate accumulation and marathon running performance. International Journal of Sports Medicine, Suttgart, v.2, p.23-36, 1981.

STAPELFELDT, B.; SCHWIRTZ, A.; SCHUMACHER, Y.O.; HILLEBRECHT, M. Workload demands in mountain bike racing. International Journal of Sports Medicine, Suttgart, v.18, p.294-300, 2004.

TAYLOR, H.L.; WANG, Y. ROWELL, L. BLOMQVIST, G. The standartization and interpretation of submaximal and maximal tests of working capacity. Pediatrics, Springfield, v.32, p.703 -22, 1963.

UNION CYCLIST INTERNATIONAL (UCI). The hub [online]. Disponível em: <http://www.uci.ch>. Acesso em: 01 jun. 2008.

VOGT, S.; HEINRICH, L.; SCHUMACHER, Y.O.; BLUM, A.; ROECKER, K.; DICKHUTH, H.H.; SCHMID, A. Power output during stage raging in professional road cycling. Medicine and Science in Sports and Exercise, Madison, v.38, n.1, p.147-51, 2006.

WILBER, R.L.; ZAWADZI, K.M.; KEARNEY, J.T.; SHANNON, M.P.; DISALVO, D. Physiological profiles of elite offroad and road cyclists. Medicine and Science in Sports and Exercise, Madison, v.29, p.1090-4, 1997.

ENDEREÇO

Vitor Pereira Costa

Centro de Educação Superior da Região Sul

Universidade do Estado de Santa Catarina

Av. Colombo Machado Salles, s/n.

88790-0oo - Laguna - SC - BRASIL

e-mail: costavp2@yahoo.com.br
Recebido para publicação: 20/01/2009

Revisado em: 13/04/2010

Aceito: 22/04/2010 